Western General Hospital, Crewe Road, Edinburgh, UK;

${ }^{3}$ Gastrointestinal Unit, Western General Hospital, Edinburgh, UK

${ }^{*}$ Correspondence: Dr A Tenesa, E-mail: albert.tenesa@ed.ac.uk.

\section{References}

1 Daly MJ, Pearce AV, Farwell L et al: Association of DLG5 R30Q variant with inflammatory bowel disease. Eur J Hum Genet 2005; 13: $835-839$.

2 Stoll M, Corneliussen B, Costello CM et al: Genetic variation in DLG5 is associated with inflammatory bowel disease. Nat Genet 2004; 36: 476-480.

3 Noble CL, Nimmo ER, Drummond $\mathrm{H}$ et al: DLG5 variants do not influence susceptibility to inflammatory bowel disease in the Scottish population. Gut 2005; 54: 1416-1420.

\title{
Reply to Tenesa et al 'Association of DLG5 and inflammatory bowel disease across populations'
}

European Journal of Human Genetics (2006) 14, 260-261. doi:10.1038/sj.ejhg.5201519; published online 4 January 2006

Tenesa et al raise an important issue regarding the involvement of DLG5 in IBD. Collectively, the heterogeneity of results at DLG5 is striking and we offer the following points for further discussion.

Point 1: In Daly et al (EJHG, 2005), we identified (and thus obviously concur with) the observation of heterogeneity among the studies we had aggregated - the overall combined data suggesting a small positive result and the fact that we confirmed a small positive effect in the additional family-based sample not included in this metaanalysis suggesting there is more than just noise here but additional studies will hopefully clarify. We are continuing to explore the source of this heterogeneity and feel that factors such as gender and ascertainment differences (which could lead to unexamined phenotypic differences) should be explored as proximal potential sources of the heterogeneity.

Point 2: We concur with the observation of heterogeneity of allele frequency across populations - it is not clear how unusual this observation is but several rarer coding polymorphisms and disease causing mutations have been noted to vary in frequency across European populations, including the most obvious comparison. The CARD15 mutations, which unequivocally confer that risks do appear to vary in frequency across European populations (for example in the paper by members of this correspondance in August 2004 Genes and Immunity).

Point 3: Here we have a slight difference in interpretation - a difference in population allele frequency does not have any directional or obligatory relationship to the risk conferred by that genotype. In this case, we have population samples that carry the allele in question between 6 and $12 \%$ or thereabouts - but whether the allele confers risk in each population is a completely independent question. While the estimated ORs could change very slightly, the heterogeneity observed is profound and ranges from showing quite significant association (Germany and Italy) to two that show none or a deficit of the risk allele among cases (UK and Scotland). A population frequency difference under any mode of inheritance cannot resolve these differences by itself although the population frequency difference could be an indirect marker for an ascertainment or population admixture difference that could be relevant.

The point that this commentary identifies that putting studies together is dangerous because different population frequencies can compromise accurate estimates since ORs can vary slightly is valid, and appropriate methods for evaluating association in the presence of heterogeneity should always be at hand. However, the implication that that is all that is needed to resolve the heterogeneity of these DLG5 studies is not supported by the analyses performed to date.

Further exploration of the source of heterogeneity at DLG5 is clearly warranted and Tenesa et al are right to highlight this fact - two studies (the German replication sample and Italy/Canada sample from Daly et al) show $P$-values of $\sim 0.001$ and two others from the UK and Scotland show no effect at all. As these are not simply cases of small sample discordancy but a highly significant discrepancy between studies that might have reasonably been considered to be addressing the same question, there is likely more to this story than has currently been discovered!

Best wishes 
Mark J Daly*,1 and John D Rioux ${ }^{1}$

${ }^{1}$ Medical and Population Genetics, The Broad Institute of the Massachusetts Institute of Technology and Harvard, Cambridge, MA, USA
${ }^{*}$ Correspondence: MJ Daly, Medical and Population Genetics, The Broad Institute of the Massachusetts Institute of Technology and Harvard, One Kendall Square, Bldg. 300, Cambridge, MA 2130, USA. Tel: + 1617252 1931; Fax: + 1617258 6505; E-mail:mjdaly@chgr.mgh.harvard.edu

\title{
Reply to Daly and Rioux response
}

\author{
European Journal of Human Genetics (2006) 14, 261. doi:10.1038/sj.ejhg.5201520; published online 4 January 2006
}

We agree with most of the points raised by Daly et al in their response to our letter. However, we have some further remarks.

Firstly, there are a number of explanations for the observed heterogeneity across the study populations. We did indeed imply that differences in allele frequencies between populations is one plausible explanation for this observation, because the estimated ORs were based on the allelic frequency in the different populations of cases and controls. The underlying reason for these differences is another issue and might be (as proposed by Daly et al) due to ascertainment differences between studies, unaccounted for environmental factors and differences in the proportion of females/males in the different studies. We also suggest that these differences might arise because of population-specific effects, since the 95\% confidence intervals (CI) for the OR in two cohorts that show an effect (German and Canada/Italy; 95\% CI are 1.16-2.03 and 1.21-3.16, respectively) do not overlap with the $95 \%$ CI for the OR in the Scottish population (95\% CI is $0.62-1.16$ ).

Secondly, another explanation for the observed heterogeneity is that the R30Q is not actually the variant influencing the phenotype. R30Q may be closely linked to the disease variant but the recombination history of each population has eroded the association in different ways.

In summary, we are in complete agreement with Daly et al that there is substantial heterogeneity in the populations studied, and the source of this heterogeneity will be of considerable interest to explore.

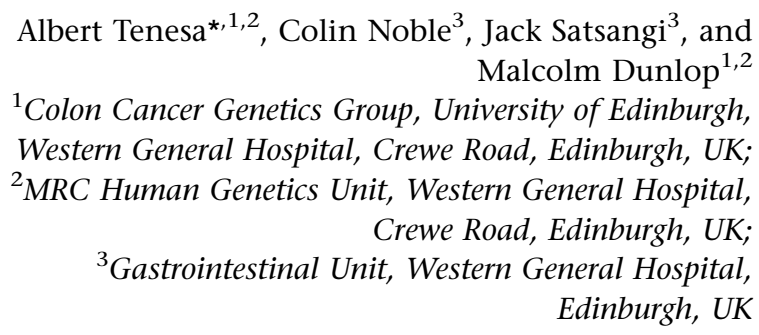

*Correspondence: A Tenesa, School of Molecular and Clinical Medicine, The University of Edinburgh, Colon Cancer Genetics Group, 4th Floor, MRC Human Genetics Unit, Western General Hospital, Crewe Road South, Edinburgh EH4 2XU, UK. Tel: + 44131 3322471; Fax: + 44131467 8450; E-mail: albert.tenesa@ed.ac.uk 